\title{
Rapid evolutionary divergence of diploid and allotetraploid Gossypium mitochondrial genomes
}

\author{
Zhiwen Chen ${ }^{1}$, Hushuai Nie', Yumei Wang ${ }^{2}$, Haili Pei ${ }^{1}$, Shuangshuang Li $^{1}$, Lida Zhang ${ }^{3}$ and Jinping Hua ${ }^{1 *}$
}

\begin{abstract}
Background: Cotton (Gossypium spp.) is commonly grouped into eight diploid genomic groups and an allotetraploid genomic group, AD. The mitochondrial genomes supply new information to understand both the evolution process and the mechanism of cytoplasmic male sterility. Based on previously released mitochondrial genomes of $G$. hirsutum $\left(A D_{1}\right)$, G. barbadense $\left(A D_{2}\right), G$. raimondii $\left(D_{5}\right)$ and $G$. arboreum $\left(A_{2}\right)$, together with data of six other mitochondrial genomes, to elucidate the evolution and diversity of mitochondrial genomes within Gossypium.

Results: Six Gossypium mitochondrial genomes, including three diploid species from $D$ and three allotetraploid species from $A D$ genome groups ( $G$. thurberi $D_{1}$, G. davidsonii $D_{3-d}$ and $G$. trilobum $D_{8} ; G$. tomentosum $A_{3}$, G. mustelinum $A_{4}$ and $\mathrm{G}$. darwinii $\mathrm{AD}_{5}$ ), were assembled as the single circular molecules of lengths about $644 \mathrm{~kb}$ in diploid species and $677 \mathrm{~kb}$ in allotetraploid species, respectively. The genomic structures of mitochondrial in D group species were identical but differed from the mitogenome of $G$. arboreum $\left(A_{2}\right)$, as well as from the mitogenomes of five species of the AD group. There mainly existed four or six large repeats in the mitogenomes of the A + AD or D group species, respectively. These variations in repeat sequences caused the major inversions and translocations within the mitochondrial genome. The mitochondrial genome complexity in Gossypium presented eight unique segments in D group species, three specific fragments in A + AD group species and a large segment (more than $11 \mathrm{~kb}$ ) in diploid species. These insertions or deletions were most probably generated from crossovers between repetitive or homologous regions. Unlike the highly variable genome structure, evolutionary distance of mitochondrial genes was 1/6th the frequency of that in chloroplast genes of Gossypium. RNA editing events were conserved in cotton mitochondrial genes. We confirmed two near full length of the integration of the mitochondrial genome into chromosome 1 of $\mathrm{G}$. raimondii and chromosome A03 of G. hirsutum, respectively, with insertion time less than 1.03 MYA.
\end{abstract}

Conclusion: Ten Gossypium mitochondrial sequences highlight the insights to the evolution of cotton mitogenomes.

Keywords: Mitochondrial genomes, Comparative genomics, Multiple DNA rearrangement, Unique segments, Repeat sequences, Gossypium

\section{Background}

Plant mitochondrial genomes (mtDNA) embrace notable characteristics, such as an extreme and highly diverse mitochondrial genome structure [1-4]. Plant mitochondrial genomes also possess highly branched and sigma-like structures [5-7] as well as multichromosomal genomes

\footnotetext{
*Correspondence: jinping_hua@cau.edu.cn

'Laboratory of Cotton Genetics, Genomics and Breeding /Key Laboratory of Crop Heterosis and Utilization of Ministry of Education/Beijing Key Laboratory of Crop Genetic Improvement, College of Agronomy and Biotechnology, China Agricultural University, Beijing 100193, China

Full list of author information is available at the end of the article
}

recently identified in three distantly-related angiosperm lineages [8-10]. The mitochondrial genome in plants is also noteworthy in that there is large variation in genome size (ranging from $\sim 66 \mathrm{~kb}$ to $11.3 \mathrm{Mb}$ ) $[8,11]$ with highly variable intergenetic regions and a considerable proportion of repeated sequences [12], frequent rearrangements [13], massive gene loss [14], and frequent endogenous and foreign DNA transfer [15-17].

In terms of structure, angiosperm mitochondrial genomes are typically mapped as circular molecules with one or more larger $(>1 \mathrm{~kb})$ repetitive sequences, which promote active homologous inter- and intra-genomic recombination 
$[4,18,19]$. However, it is not clear how plant mitochondrial genomes rearrange so frequently or how the genome sizes can vary dramatically over relatively short evolutionary period. This dynamic organization of the angiosperm mitochondrial genome provides unique information as well as an appropriate model system for studying genome structure and evolution. More syntenic sequences will be helpful to interpret the evolutionary processes for diverse angiosperm mitochondrial structures.

Cotton (Gossypium) is the most important fiber crop plant in the world [20]. Four domesticated species remain as cultivated crops: the New World allopolyploid species G. hirsutum and G. barbadense $(2 \mathrm{n}=52)$, and the Old World diploid species G. arboreum and G. herbaceum $(2 \mathrm{n}=26)[20,21]$. The primary cultivated one is Upland cotton (G. hirsutum L.), accounting for more than $90 \%$ of global cotton fiber output. Gossypium includes 52 species: seven allotetraploid species and 45 diploids [21, 22]. The nascent allopolyploid species spread throughout the American tropics and subtropics, diverging into at least seven species, namely, G. hirsutum $\mathrm{L}$. $\left(\mathrm{AD}_{1}\right), G$. barbadense L. $\left(\mathrm{AD}_{2}\right)$, G. tomentosum Nuttalex Seemann $\left(\mathrm{AD}_{3}\right)$, G. mustelinum Miersex Watt $\left(\mathrm{AD}_{4}\right)$, G. darwinii Watt $\left(\mathrm{AD}_{5}\right)$, G. ekmanianum $\left(\mathrm{AD}_{6}\right)$, and G. stephensii $\left(\mathrm{AD}_{7}\right)$ [20-22]. The diploid Gossypium species comprise eight monophyletic genome groups, A, B, C, D, E, F, $G$ and $K$ group [20, 23]. With the rapid development of next-generation sequencing technologies [24, 25], cotton genomics research has rapidly progressed in recent years, such that nuclear genome sequences have now been published for model diploids ( $\mathrm{D}_{5}$-genome $[26,27], \mathrm{A}_{2}$-genome [28]), and for the allopolyploids $\left(\mathrm{AD}_{1}-\mathrm{G}\right.$. hirsutum $[29,30]$, $\mathrm{AD}_{2}$-G. barbadense [31, 32]). In addition, a large number of Gossypium organelle genome sequences have been released [33-39]. Compared to the highly conserved chloroplast genome structures [34-36], comparative analysis revealed rapid evolutionary divergence of Gossypium mitochondrial genomes [37-39], which proved that deep analyses of more mitochondrial genomes would provide new data to consider the evolutionary relationships and to explore the mechanism of cytoplasmic male sterility (CMS).

Cytoplasmic male sterility (CMS) is a maternallyconferred reproductive trait that relies on the expression of CMS-inducing mitochondrial sequences [40]. Many examples of CMS stem from the consequences of recombination [40-42]. Often, these chimeric CMS genes exhibit co-transcription with upstream or downstream functional genes, which typically affect the mitochondrial electron transfer chain pathways to fail to produce functional pollen [43]. Rearrangements in the mitochondrial DNA involving known mitochondrial genes as well as unknown sequences result in the creation of new chimeric open reading frames, which encode proteins containing transmembrane and lead to cytoplasmic male sterility by interacting with nuclear-encoded genes [43-45].

Here, six Gossypium mitochondrial genomes are reported, including three diploid species from $\mathrm{D}$ genome groups (G. thurberi $\mathrm{D}_{1}$, G. davidsonii $\mathrm{D}_{3-\mathrm{d}}$ and G. trilobum $\mathrm{D}_{8}$ ) and three allotetraploid species from $\mathrm{AD}$ genome groups (G. tomentosum $\mathrm{AD}_{3}, G$. mustelinum $\mathrm{AD}_{4}$ and $G$. darwinii $\left.\mathrm{AD}_{5}\right)$. Comparative mitochondrial genome analysis then revealed rapid mitochondrial genome rearrangement and evolution between diploid and allotetraploid Gossypium. In addition, one of the most surprising outcomes of comparative analyses is how rapidly mitochondrial sequence segments altered within a single subspecies. Finally, the four mitogenomes of D group species provided the useful data resources for interpreting the CMS-related genes in G. trilobum $\mathrm{D}_{8}$ cotton.

\section{Methods}

Plant materials and mitochondrial DNA extraction

Seeds of diploid and allotetraploid Gossypium species were acquired from the nursery on the China National Wild Cotton Plantation in Sanya, Hainan, China. Mitochondria were isolated from week-old etiolated seedlings, and the mitochondrial DNA samples were extracted from an organelle-enriched fraction isolated by differential and sucrose gradient centrifugation, essentially as described earlier [37-39, 46].

\section{Mitochondrial genome sequencing and primary data processing}

A total of $\sim 5$ million clean paired-end reads were sequenced from a $~ 500$ bp library for each of three diploid species, respectively. We produced $300 \mathrm{bp}$ read length with paired-end sequencing, using MiSeq sequencing method on Illumina platform at Beijing Biomarker Technologies Co, LTD. A total of $\sim 11$ million clean paired-end reads were sequenced from a $\sim 500$ bp library with paired-end, $300 \mathrm{bp}$ read length, for each of three allotetraploid species, respectively, using the same method. Raw sequences were first evaluated by two quality control tools, Trimmomatic [47] and FilterReads module in Kmernator ([https://github. com/JGI-Bioinformatics/Kmernator]) to remove any potential undesirable artifacts in the data such as adapters or low quality or "N" bases and so on.

\section{Genomes assembly and sequence verification}

Six Gossypium draft mitogenomes were assembled de novo from the clean reads with velvet 1.2.10 [48] or combining FLASH [49] and Newbler (Version 2.53) methods, respectively. For the first assembly method, i.e., the 300-bp paired-end reads from six Gossypium species, we performed multiple velvet runs with different combinations of kmer values (for $(\mathrm{kmer}=75$; $\mathrm{kmer}<=209$; $\mathrm{kmer}=\mathrm{kmer}+2),(42$ in total)). Three Kmer values (193, 195, 197), owning larger 
N50 values, less contig number, were used to assemble the mitogenomes. For each velvet run, the minimum coverage parameter was set to $10 \times$ and scaffolding was turned off when the data sets contained paired-end reads. For each of assembly, mitochondrial contigs were identified by blastn [50] searches with known Gossypium mitochondrial genomes for scaffolding and gap filling [37-39]. The best draft assemblies for six Gossypium were chosen as the assembly that maximized total length of mitochondrial contigs after combining three Kmer values assembly. In another assembly method, we combined FLASH [49] and Newbler (Version 2.53) softwares together. First, FLASH provides the use of paired-end libraries with a fragment size (500 bp) shorter than twice the read length (300 bp) an opportunity to generate much longer reads ( $500 \mathrm{bp}$ ) by overlapping and merging read pairs [49]. The merging file was then assembled using Newbler (Version 2.53) software. Finally, the assembled mitochondrial scaffolds were aligned with known Gossypium mitochondrial genomes [37-39] for anchoring scaffold directions and gap filling. Thus, we combined two types of the assembly results to complete the six Gossypium mitogenomes. The final remaining gaps were filled by aligning individual pair-end sequence reads that overlapped the scaffolds or contig ends using Burrows-Wheeler Aligner (BWA 0.7.10-r789) software [51].

To evaluate six mitogenomes sequence assembly quality and accuracy, pair-end reads were mapped onto their respective consensus sequences with BWA 0.7.10-r789 [51]. The BWA mapping resulting SAM files were transformed into BAM files using samtools view program [52]. The BWA mapping results for these pair-end reads in BAM files were then used to calculate depth of sequencing coverage through samtools depth program [52]. For all six Gossypium species, the Illumina reads covered all parts of the genome consistently, with the average coverage ranging from $50 \times$ to $200 \times$.

\section{Genome annotations and sequence analyses}

Gossypium mitochondrial genes from the six species were annotated using G. hirsutum and G. barbadense mitogenomes as references. Functional genes (other than tRNA genes) were identified by local blast searches against the database, whereas tRNA genes were predicted de novo using tRNA scan-SE [53]. Repeat-match program in MUMmer [54] was used to identify repeated sequences within six Gossypium mitogenomes. Their genome maps were generated using OGDRAW [55] and the repeat map was drawn by Circos [56].

Collinear blocks were generated among the ten mitochondrial genomes of Gossypium using the progressiveMauve program [57]. To determine the amount of Gossypium mitochondrial genome complexity shared between species, each pair of mitogenomes was aligned using blastn
[50] with an e-value cutoff of $1 \times 10^{-5}$. Using these parameters, the blastn searches should be able to detect homologous sequences as short as $30 \mathrm{bp}$. The unique segments in Gossypium mitogenomes identified in this study were summarized as follows: i) Paired-end reads were mapped onto their respective consensus sequences using the BurrowsWheeler Aligner (BWA 0.7.10-r789) software [51]; ii) The BWA mapping resulting SAM files were transformed into BAM files using the samtools program [52] set to the default parameters; and iii) Structure variations (SVs) and InDels reported in this work were manually visualized using the Integrative Genomics Viewer (IGV) software [58].

\section{RNA editing identification}

RNA edit sites were computationally predicted using the batch version of the PREP-Mt. online server [59], with a cutoff value of 0.2 .

\section{Phylogenetic analyses and estimation of evolutionary divergence}

For phylogenetic analyses, 36 protein-coding genes were extracted from 10 Gossypium species and two outgroups: C. papaya and A. thaliana. Sequence alignments for 36 concatenated genes, each chloroplast and mitochondrial coding exons were carried out by MAFFT [60]. Phylogenetic analyses were performed with the same methods to our previous studies [35, 36, 39]. P-distances for chloroplast and mitochondrial coding genes were calculated with MEGA5.05 [61].

\section{Identifying nuclear mtDNAs in Gossypium, estimation of evolutionary divergence and divergence time between mitochondrial sequences and numts}

Dot matrix comparisons were generated between the mitochondrial and nuclear chromosomes of four Gossypium species using the nucmer program of MUMmer with the parameters 100-bp minimal size for exact match and 500-bp minimal interval between every two matches [54]. The detailed comparison results were shown in Fig. 7: G. raimondii mitochondrial [39] and nuclear chromosomes [27] in Fig. 7a, G. arboreum mitochondrial [39] and nuclear chromosomes [28] in Fig. 7b, G. hirsutum mitochondrial [37] and nuclear chromosomes [30] in Fig. 7c, G. barbadense mitochondrial [38] and nuclear chromosomes [32] in Fig. 7d. Sequence alignments for each coding, intronic, and intergenic spacer regions were carried out by MAFFT [60] software. P-distances between mitochondrial sequences and numts were calculated with MEGA5.05 [61]. In order to estimate how old these insertions are, $\mathrm{p}$-distance rates and some estimates of rate/million years were studied here. The divergence time of between mitochondrial native sequences and numts was calculated by the following Formula: $\mathrm{T}=\mathrm{p}$-distance $/\left(\mathrm{r}_{\mathrm{nu}}+\mathrm{r}_{\mathrm{mt}}\right)$ [62]. Based on Gaut et al. (1996) and Muse et al. (2000), the $r_{n u}$ and $r_{m t}$ values 
were estimate as $r_{n u}=6.5 \times 10^{-9}$ and $r_{\mathrm{mt}}=2 \times 10^{-10}$, respectively $[63,64]$. It also has to be made clear that the underlying assumption is homogeneity in rate since their divergence from a common ancestor.

\section{Results}

\section{Gossypium mitochondrial genomes from diploid and} allotetraploid species

Six Gossypium mitochondrial genomes were obtained in present study, including three diploid D species and three allotetraploid AD species. Complete mitochondrial DNA sequences were deposited in the GenBank database respectively: G. thurberi $\mathrm{D}_{1}$ (Accession No. KR736343), G. davidsonii $\mathrm{D}_{3-\mathrm{d}}$ (Accession No. KR736344), G. trilobum $\mathrm{D}_{8}$ (Accession No. KR736346), G. tomentosum $\mathrm{AD}_{3}$ (Accession No. KX388135), G. mustelinum $\mathrm{AD}_{4}$ (Accession No. KX388136) and G. darwinii $\mathrm{AD}_{5}$ (Accession No. KX388137) (Table 1). The six Gossypium mitogenomes were all assembled as single circular molecules of lengths about $644 \mathrm{~kb}$ in diploid (Additional file 1: Figure S1A) and $677 \mathrm{~kb}$ in allotetraploid (Additional file 1: Figure S1B), respectively. The genomic structures were identical within diploid group (Fig. 1) and allotetraploid group (Fig. 2), respectively, but differed between the two groups. The diploid group had six large repeats $(>1 \mathrm{~kb})$ whereas the allotetraploid group had four large repeats (Fig. 1; Fig. 2), which may be involved in the rearranged mitogenome organizations in diploid and allotetraploid Gossypium.

Comparison of mitochondrial gene content among Gossypium species reveals a conserved pattern of evolutionary stasis for diploid and allotetraploid species, respectively (Table 1). The Gossypium mitogenomes contain 36 protein coding genes with five genes (rps1, rps2, rps11, rps13 and rps19) being lost during coevolution with nucleus, compared to the common ancestor of seed plants $[65,66]$. The repeat sequences confer some redundant gene copies ( $n a d 4$, nad9 and $m t t B$ ) in three allotetraploid species with uncertain functions (Additional file 2: Table S1). These mitochondrial genomes (Table 1) show high identity in gene content but no similarity in genome organization (Fig. 1; Fig. 2) with each other or with previously published cotton mitochondrial genomes [37-39], with apparent major differences in genome organization and size.

\section{Syntenic regions and rearrangement}

After combining four other Gossypium mitochondrial genomes [37-39], totally, ten species of five allotetraploid and five diploid were used for analyses: one from A genome, four from $\mathrm{D}$ genome and five from $\mathrm{AD}$ genome groups, respectively (Table 1 ). Syntenic regions were identified between ten Gossypium mitochondrial genomes with eight large major syntenic blocks (Fig. 3). The genomic structures were totally identical in four species of D group, indicating that the mitochondrial genome structures may be highly conserved in D genome species. G. trilobum $\left(\mathrm{D}_{8}\right)$ contributed the cytoplasmic male sterility (CMS) cytoplasm in cotton [67-69], however, no genome rearrangement or large indel segments variations compared with mito-genomes of other D species, implying that the mitochondrial CMS-associated gene in cotton may function with different mechanism.

In addition, compared to $\mathrm{D}$ group species, the mitochondrial genomic structure in A group $\left(\mathrm{A}_{2}\right)$ was highly rearranged (Fig. 3). Interestingly, genome rearrangements also occurred among the five allotetraploid species, as was already reported in the G. hirsutum - G. barbadense comparison [38]. Despite the fact that three allotetraploid

Table 1 Main features of the ten assembled Gossypium mitogenomes

\begin{tabular}{|c|c|c|c|c|c|c|c|c|c|c|}
\hline $\begin{array}{l}\text { Genome } \\
\text { Characteristics }\end{array}$ & $\begin{array}{l}\text { G. } \\
\text { thurberi }\end{array}$ & $\begin{array}{l}\text { G. } \\
\text { davidsonii }\end{array}$ & $\begin{array}{l}\text { G. } \\
\text { raimondii }\end{array}$ & $\begin{array}{l}\text { G. } \\
\text { trilobum }\end{array}$ & $\begin{array}{l}\text { G. } \\
\text { arboreum }\end{array}$ & $\begin{array}{l}\text { G. } \\
\text { hirsutum }\end{array}$ & $\begin{array}{l}\text { G. } \\
\text { barbadense }\end{array}$ & $\begin{array}{l}\text { G. } \\
\text { tomentosum }\end{array}$ & $\begin{array}{l}\text { G. } \\
\text { mustelinum }\end{array}$ & $\begin{array}{l}\text { G. } \\
\text { darwinii }\end{array}$ \\
\hline Accession & KR736343 & KR736344 & KR736345 & KR736346 & KR736342 & JX065074 & KP898249 & KX388135 & KX388136 & KX388137 \\
\hline Genome groups & $D_{1}$ & $D_{3-d}$ & $\mathrm{D}_{5}$ & $D_{8}$ & $\mathrm{~A}_{2}$ & $A D_{1}$ & $A D_{2}$ & $\mathrm{AD}_{3}$ & $\mathrm{AD}_{4}$ & $\mathrm{AD}_{5}$ \\
\hline Genome size (bp) & 644,395 & 644,311 & 643,914 & 644,460 & 687,482 & 621,884 & 677,434 & 677,295 & 677,306 & 677,286 \\
\hline Circular chromosomes & 1 & 1 & 1 & 1 & 1 & 1 & 1 & 1 & 1 & 1 \\
\hline Percent $\mathrm{G}+\mathrm{C}$ content & 44.92 & 44.94 & 44.94 & 44.92 & 44.94 & 44.95 & 44.98 & 44.98 & 44.98 & 44.97 \\
\hline Protein genes & 36 & 36 & 36 & 36 & 39 & 36 & 39 & 39 & 39 & 39 \\
\hline tRNA genes & 27 & 27 & 27 & 27 & 30 & 29 & 30 & 30 & 30 & 30 \\
\hline Native & 17 & 17 & 17 & 17 & 17 & 17 & 17 & 17 & 17 & 17 \\
\hline Plastid-derived & 10 & 10 & 10 & 10 & 13 & 12 & 13 & 13 & 13 & 13 \\
\hline tRNAs with introns & 3 & 3 & 3 & 3 & 4 & 3 & 4 & 4 & 4 & 4 \\
\hline rRNA genes & $7^{*}$ & $7^{*}$ & $7^{*}$ & $7^{*}$ & $7^{*}$ & 4 & 6 & 6 & 6 & 6 \\
\hline $\begin{array}{l}\text { Large repeats: } \\
>1 \text { kb(number) }\end{array}$ & 6 & 6 & 6 & 6 & 4 & 4 & 4 & 4 & 4 & 4 \\
\hline
\end{tabular}




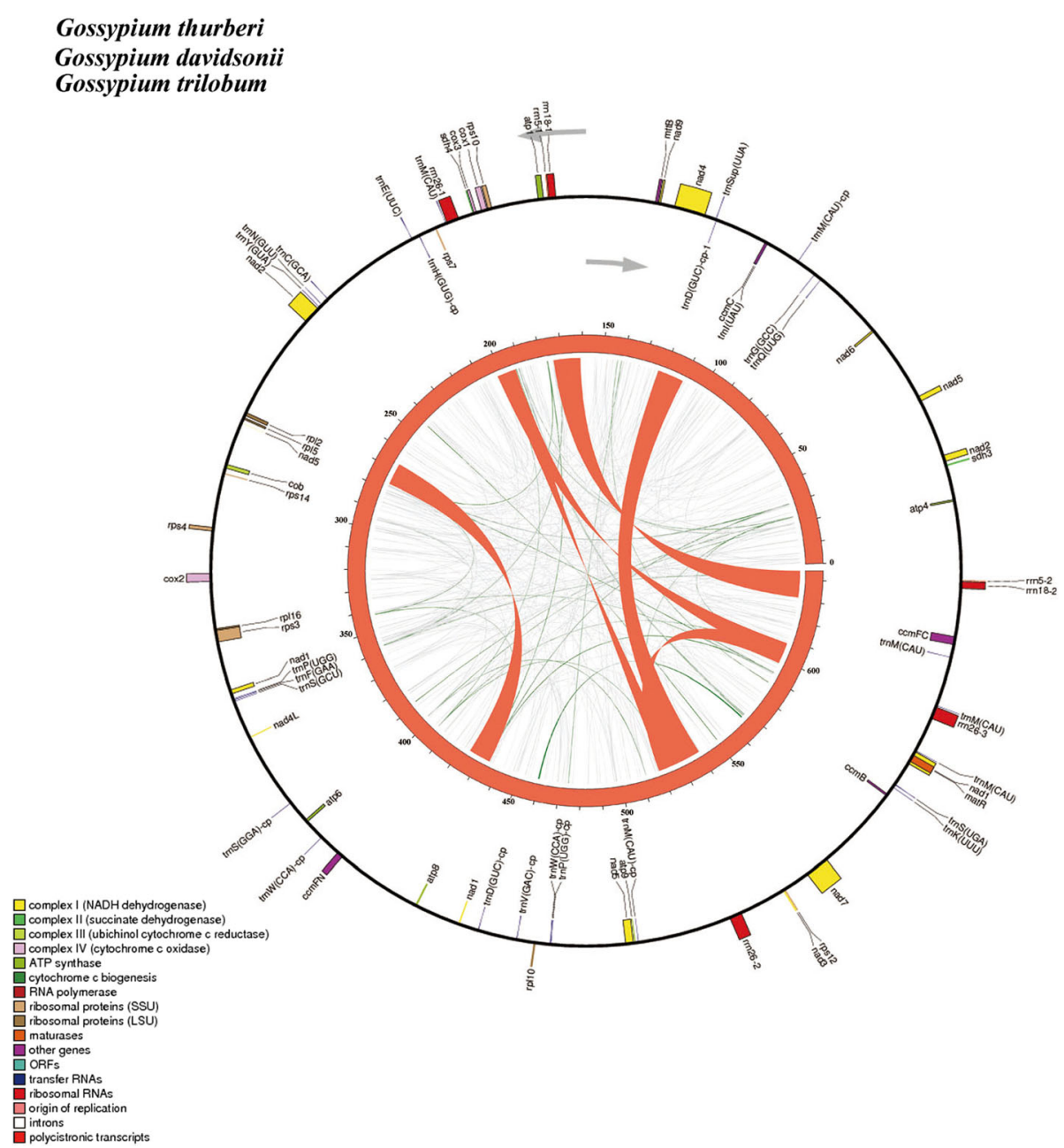

Fig. 1 Genome maps of three diploid Gossypium mitogenomes. The map shows both the gene map (outer circle) and repeat map (inner map). Genes exhibited on the inside of outer circles are transcribed in a clockwise direction, while genes on the outside of outer circles are transcribed in a reverse direction. The inner circle reveals the distribution of repeats in two mitogenomes with curved lines and ribbons connecting pairs of repeats and width proportional to repeat size. The red ribbons represent $>=1 \mathrm{~Kb}$ repeats, the very deep green lines represent repeats between $100 \mathrm{bp}$ to $1 \mathrm{~Kb}$ and the very light grey lines represent repeats <100 bp. The numbers give genome coordinates in kilobase

species (G. tomentosum $\mathrm{AD}_{3}$, G. mustelinum $\mathrm{AD}_{4}$ and G. darwinii $\mathrm{AD}_{5}$ ) exhibited the same genome organization, a disorder existed between the mitogenomes of G. hirsutum $\mathrm{AD}_{1}$ and G. barbadense $\mathrm{AD}_{2}$ (Fig. 3).

\section{Gene order and repeat sequences}

To uncover the formation mechanism of recombination generating multiple genomic arrangements in Gossypium, we presented the gene order with five major linear models and genes located in repeat regions shown in bold (Additional file 3: Figure S2). The gene orders in the D genome species are highly conserved but not identical to that in either $G$. arboreum $\left(\mathrm{A}_{2}\right)$ or the $\mathrm{AD}$ groups with six-seven gene clusters scattered. Though there exists a few changes in mitochondrial gene order within each of five models in the three Gossypium lineages as shown by ten released mitogenomes, a minimum of two and three changes (inversions and translocations) need to be invoked to explain the differences of gene order among diploid and allotetraploid Gossypium, respectively (Fig. 3), and how these genomic rearrangements events happened are difficult to reconstruct. Repeat sequences have been suggested to serve as sites of homologous recombination, resulting in gene order changes in mitochondrial genomes [19].

The repeat sequences detected in the Gossypium mitogenomes in present and earlier studies [37-39] may be responsible for mitochondrial gene order changes between diploids and allotetraploids (Table 2). There mainly existed six or four large repeats in $\mathrm{D}$ group or $(\mathrm{A}+\mathrm{AD})$ groups, respectively. The repeat sizes were almost identical in $\mathrm{D}$ group but differed in (A + AD) groups. Despite a big 


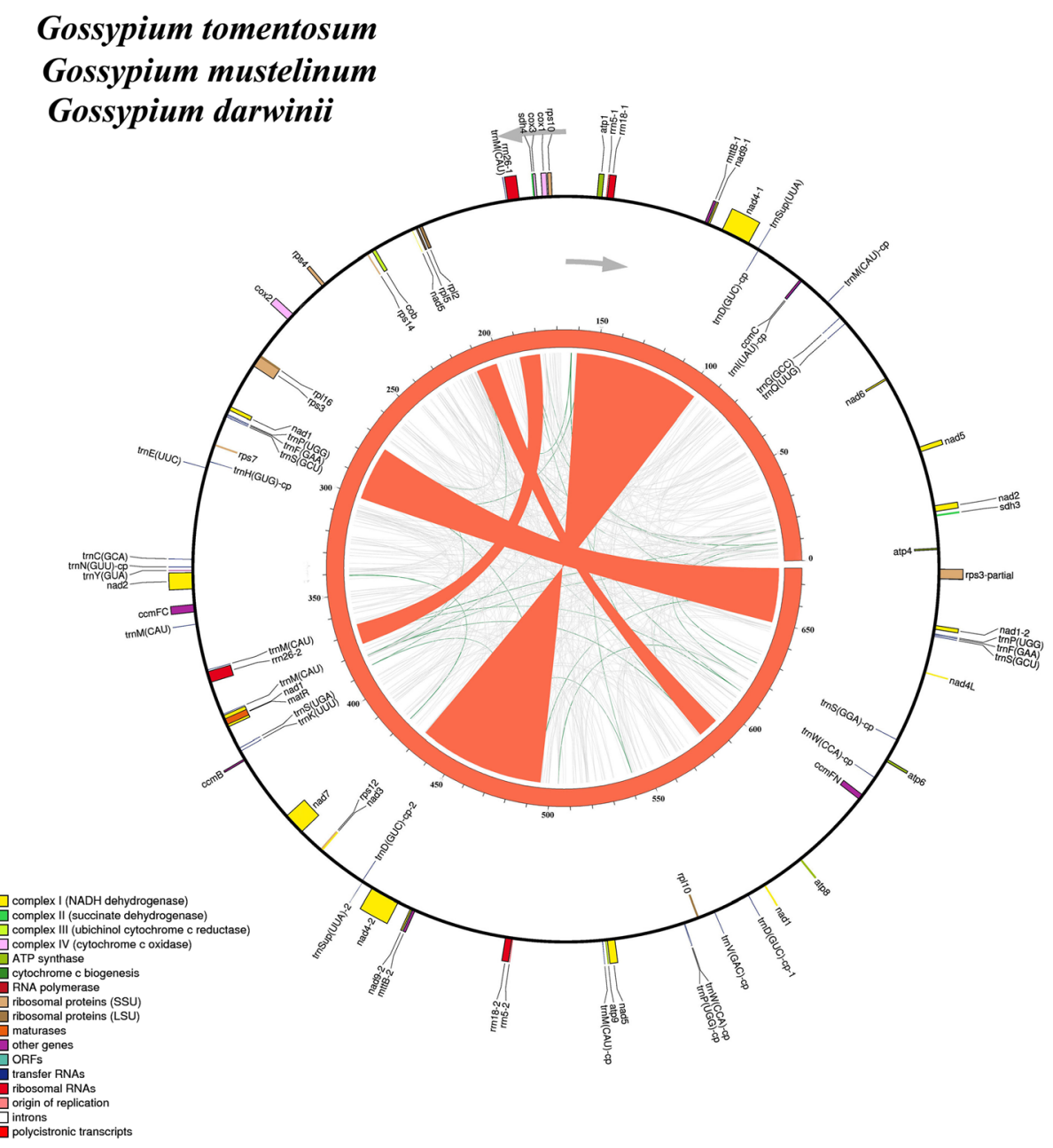

Fig. 2 Genome maps of three allotetraploid Gossypium mitogenomes. The map explanations were the same to Fig. 1

deletion, about $50 \mathrm{~kb}$ that occurred in R1 of G. hirsutum $\left(A D_{1}\right)$, a $27 \mathrm{~kb}$ repeat was unique to the $\mathrm{AD}$ group. In addition, the repeat diverged considerately between the two diploid Gossypium groups (Table 2). In addition, genes in the border of the gene clusters in Gossypium were almost located in or close to the repeat sequences (Additional file 3: Figure S2). These variations in repeat sequences may perhaps cause the major inversions and translocations within the mitochondrial genome of the common ancestor shared by D-A and A-AD species after Gossypium had diverged. Evolution of gene order in diploid D group mitogenomes of Gossypium is overall quite conservative, but exists divergence between different diploid and allotetraploid lineages.

\section{Conservation and variants in Gossypium mitochondrial genomes}

Considering that all the Gossypium mitogenomes have similar genome complexity, comparative analysis were conducted to determine the proportion of the sequences that each shared in common with the others (Table 3). One of the most surprising outcomes is how rapidly sequence segments were gained or lost. Genome specific fragments is not present in any two genomes of the D or AD groups, respectively (Table 3 ). While reciprocity is generally not seen in any other comparisons, even between the two diploid mitogenome groups: G. arboreum $\left(\mathrm{A}_{2}\right)$ lost $2.97 \%$ of the sequences present in $\mathrm{D}$ group, but $\mathrm{D}$ group lost $0.77 \%$ of the A species' sequences. G. $\operatorname{arboreum}\left(\mathrm{A}_{2}\right)$ is attributed to be the putative maternal contributor to the progenitor of $\mathrm{AD}$ group [21, 34, 70], however, each of the five $\mathrm{AD}$ group genomes has lost substantial amounts of sequence that is present in the G. arboreum $\left(\mathrm{A}_{2}\right)$ genomes and vice versa (Table 3 ). The difference is more striking when comparing mitogenomes of $\mathrm{D}$ group and $\mathrm{AD}$ group: $\mathrm{D}$ group species lost only $0.64 \%$ of the $\mathrm{AD}$ group mitogenomes, but $\mathrm{AD}$ group species lost $4.41 \%$ of the $\mathrm{D}$ group mito-genomes. Reciprocal differences were more apparent in the comparisons between male-fertile and CMS (cytoplasmic male sterility) mitochondrial genomes [71-73]. 


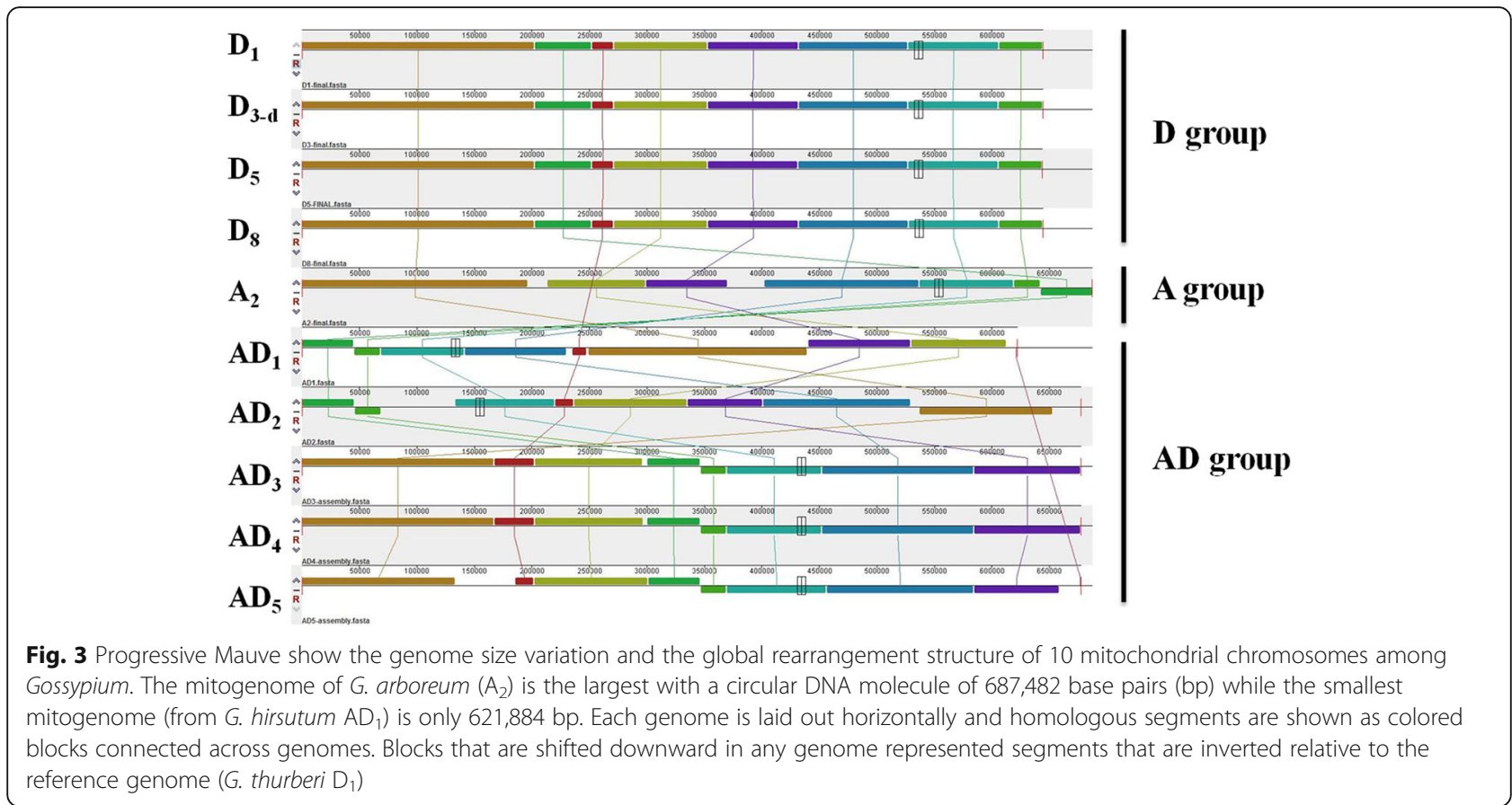

In fact, the genome complexity in Gossypium presented eight unique segments ranging from $108 \mathrm{bp}$ to $7888 \mathrm{bp}$ in length in $\mathrm{D}$ group mitochondrial genomes, comprising a total of $18,194 \mathrm{bp}$ (Indels of $<100 \mathrm{bp}$ were not included) (Fig. 4a, showing one of the unique segments); while three specific fragments detected in (A + AD) group mitochondrial genomes with the largest size 3876 bp in length, 4315 bp in total (Fig. 4b). In addition, a large segment more than $11 \mathrm{~kb}$ in length that is present in the diploid mitogenome is not present in any of the other five allotetraploid mitogenomes (Fig. 4c). Despite the fact that the ancestor of A-genome group is the maternal source of extant allotetraploid species [20-23, 34], unique presence/absence variations existed as well (Fig. 4c; Additional file 4: Figure S3).

\section{RNA editing in Gossypium mitochondrial genes}

Post-transcriptional RNA-editing of mitochondrial genes is both ubiquitous and important for regulation [74]. Typically, RNA editing of mitochondrial transcripts in flowering plants occurs in coding regions of mitochondrial transcripts to convert specific cytosine residues to uracil $(\mathrm{C} \rightarrow \mathrm{U})[75,76]$. For ten Gossypium species, we predicted sites of $\mathrm{C}$-to- $\mathrm{U}$ editing using the PREP-Mt. online tool [59] with a cutoff of 0.2. The number of predicted C-to- $\mathrm{U}$ edits across the entire coding regions of their shared 36 protein genes is almost similar for ten Gossypium species (451), with one editing site lower in G. hirsutum (450 sites) caused in nad3 gene (Table 4). The simplest interpretation of these results is that the

Table 2 Major repeat sizes (>1 kb) in Gossypium mitochondrial genomes

\begin{tabular}{llllllr}
\hline Gossypium species & Repeat 1 $(\mathrm{kb})$ & Repeat 2 $(\mathrm{kb})$ & Repeat 3 $(\mathrm{kb})$ & Repeat 4 $(\mathrm{kb})$ & Repeat 5 $(\mathrm{kb})$ & Repeat 6 $(\mathrm{kb})$ \\
\hline G. thurberi & 12,921 & 12,669 & 10,632 & 9121 & 8544 & 7317 \\
G. davidsonii & 12,937 & 12,669 & 10,624 & 9121 & 8544 & 7317 \\
G. raimondii & 12,741 & 12,670 & 10,624 & 9121 & 8544 & 7317 \\
G. trilobum & 12,921 & 12,669 & 10,650 & 9121 & 10,246 & 1317 \\
G. arboreum & 63,789 & 32,975 & 15,029 & 10,251 & 10,246 & 10,246 \\
G. hirsutum & 10,302 & 27,495 & 10,623 & 10,246 & \\
G. barbadense & 63,904 & 26,936 & 10,615 & 10,246 & \\
G. tomentosum & 63,888 & 27,425 & 10,614 & 10,614 & & \\
G. mustelinum & 63,886 & 27,425 & 27,425 & 10,614 & & \\
G. darwinii & 63,893 & & &
\end{tabular}


Table 3 Percentage of Gossypium mitochondrial genome complexity that is absent in other genomes

\begin{tabular}{llllllllllll}
\hline & $\mathrm{VS}$ & $\mathrm{D}_{1}$ & $\mathrm{D}_{3-d}$ & $\mathrm{D}_{5}$ & $\mathrm{D}_{8}$ & $\mathrm{~A}_{2}$ & $\mathrm{AD}_{1}$ & $\mathrm{AD}_{2}$ & $\mathrm{AD}_{3}$ & $\mathrm{AD}_{4}$ & $\mathrm{AD}_{5}$ \\
\hline References genomes & $\mathrm{D}_{1}$ & & 0 & 0 & 0 & 0.77 & 0.64 & 0.64 & 0.64 & 0.64 & 0.64 \\
& $\mathrm{D}_{3-d}$ & 0 & & 0 & 0 & 0.77 & 0.64 & 0.64 & 0.64 & 0.64 & 0.64 \\
& $\mathrm{D}_{5}$ & 0 & 0 & & 0 & 0.77 & 0.64 & 0.64 & 0.64 & 0.64 & 0.64 \\
& $\mathrm{D}_{8}$ & 0 & 0 & 0 & & 0.77 & 0.64 & 0.64 & 0.64 & 0.64 & 0.64 \\
& $\mathrm{~A}_{2}$ & 2.97 & 2.97 & 2.97 & 2.97 & & 0.12 & 0.12 & 0.12 & 0.12 & 0.12 \\
& $\mathrm{AD}_{1}$ & 4.41 & 4.41 & 4.41 & 4.41 & 1.63 & & 0 & 0 & 0 & 0 \\
& $\mathrm{AD}_{2}$ & 4.41 & 4.41 & 4.41 & 4.41 & 1.63 & 0 & & 0 & 0 & 0 \\
& $\mathrm{AD}_{3}$ & 4.41 & 4.41 & 4.41 & 4.41 & 1.63 & 0 & 0 & 0 & 0 \\
& $\mathrm{AD}_{4}$ & 4.41 & 4.41 & 4.41 & 4.41 & 1.63 & 0 & 0 & 0 & 0 & 0 \\
& $\mathrm{AD}_{5}$ & 4.41 & 4.41 & 4.41 & 4.41 & 1.63 & 0 & 0 & 0 & 0
\end{tabular}

Note: Small deletions ( $<100$ bp) are not considered to be missing segments. $D_{1}=G$. thurberi, $D_{3-d}=G$. davidsonii, $D_{5}=G$. raimondii, $D_{8}=G$. trilobum, $A_{2}=G$. arboreum, $\mathrm{AD}_{1}=G$. hirsutum, $\mathrm{AD}_{2}=G$. barbadense, $\mathrm{AD}_{3}=G$. tomentosum, $\mathrm{AD}_{4}=G$. mustelinum, $\mathrm{AD}_{5}=G$. darwinii

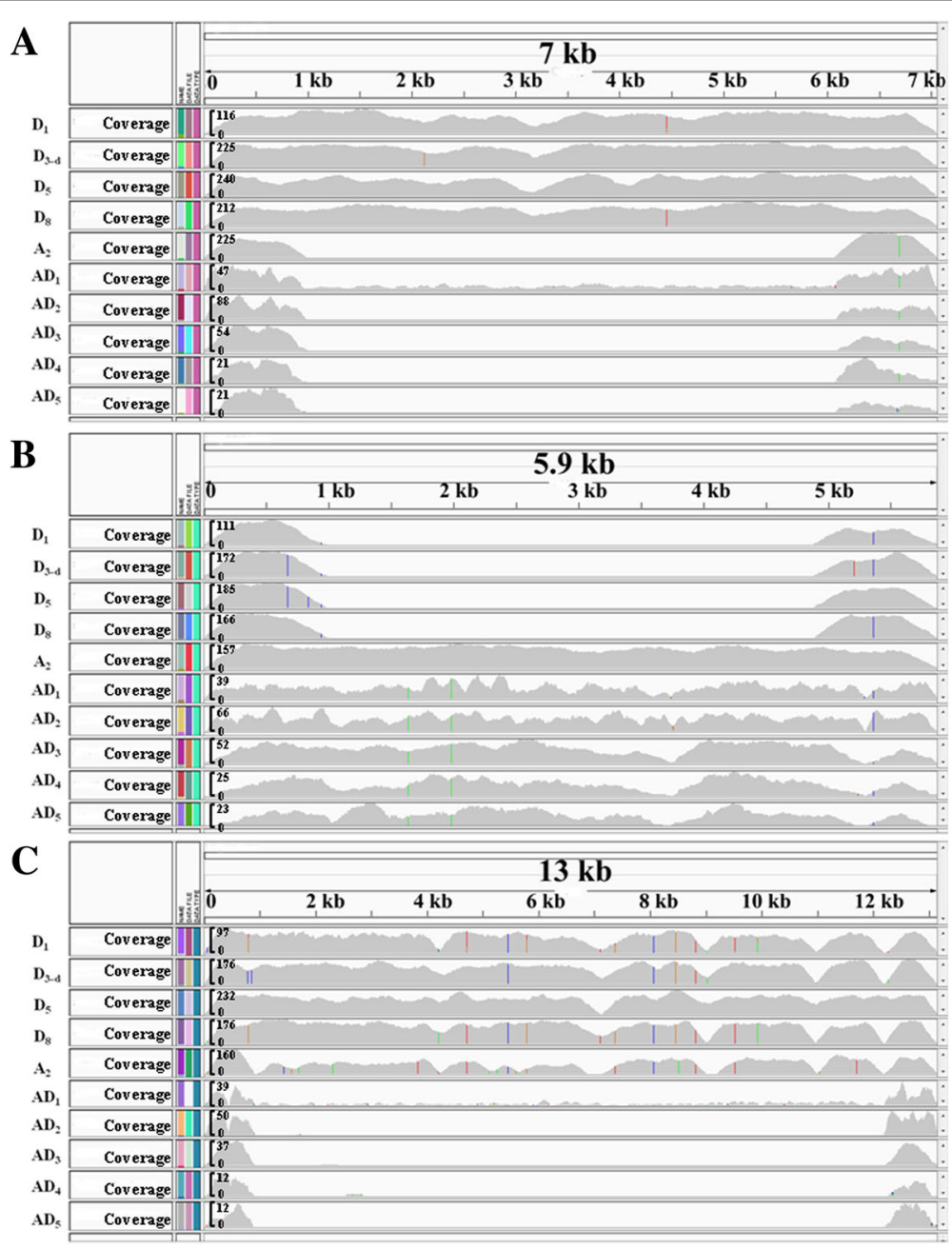

Fig. 4 Observed coverage of mapped paired-end reads supporting the existence of a large insertion or deletion in Gossypium species. IGV screenshot of the variability and coverage observed in ten Gossypium sequence samples. Upper panel represent the unique sequences coordinates. There are ten panels corresponding to the different Gossypium sequences. The track in each of these panels describes the density of read mapping or coverage depth. a: unique segment in $\mathrm{D}$ group. $\mathbf{b}$ : unique segment in $A+A D$ group. $\mathbf{c}$ : unique segment in diploid groups 
whole of edit sites in ten Gossypium species were present in their common ancestor, while the species-specific sites are less derived in cotton.

\section{Mitochondrial genome evolution in Gossypium}

Phylogenetic relationships among 10 Gossypium species with two outgroups, was generated using a concatenated analysis of 36 mitochondrial protein-coding genes (Fig. 5). The topology of the resulting tree supports G. arboreum as the maternal donor to polyploid cotton species, which further supports our former result [39]. We mapped these specific indels into the phylogenetic clades, as shown in Fig. 5, which implied an ongoing dynamic divergence process. First, eight mitogenome fragments (U1-U8) were involved in loss events after G. arboreum $\left(\mathrm{A}_{2}\right)$ diverged from a common ancestor shared with the $\mathrm{D}$ genome group. Subsequently, three genome fragments (U9-U11) were transferred from the nucleus to the mitochondrial genome in an A-genome ancestor or contributor/donator before the formation of the allopolyploidization event. Finally, a large genome fragment (U12), about $11 \mathrm{~kb}$, was lost during the divergence process of allotetraploid ancestor species (Fig. 5). This $11 \mathrm{~kb}$ deletion (corresponding to U12 in diploid mitogenomes) was adjacent to the specific repeat sequence $\mathrm{R} 2$ in $\mathrm{AD}$ group, which might lead to the formation of $\mathrm{R} 2$ repeat sequences (unique to $\mathrm{AD}$ group species) during evolution. In addition, variations in repeat and Indels lengths also cause the great difference of Gossypium mitochondrial genome sizes (Fig. 3 and Fig. 5). MtDNA intergenic regions are known to possess more unique segments than genic regions, however, shorter repeats account for the relatively small size of the D-group mitochondrial genomes. Interestingly, a large deletion $\sim 50 \mathrm{~kb}$ in length in R1 may lead to the small size of G. hirsutum $\left(\mathrm{AD}_{1}\right)$ [37], compared to the other four allotetraploid genomes. Comparative mitochondrial genome analysis revealed rapid mitochondrial genome rearrangement and evolution even within a single subspecies.

In addition, we calculated the p-distances representing evolutionary divergence from 78 chloroplast and 36 mitochondrial protein-coding exons among 10 Gossypium species, as shown in Fig. 6 . Here, the average evolutionary divergence was 0.0031 in chloroplast genes but only 0.0005 in mitochondrial genes among 10 Gossypium species. The mitochondrial genes were highly conserved with low evolutionary divergence, however, their genome structures displayed the extremely rapid evolution of various changes, including repeat and large indels variations. Based on these results, the evolutionary distance of mitochondrial genomes are much lower than the chloroplast genomes in Gossypium, however, rapid varying mitogenome structures evolves much faster than the highly conserved chloroplast genomes [35-39].

\section{MtDNAs insert into the nuclear chromosomes in Gossypium}

In this study, four sets of mitochondrial and nuclear genomes of Gossypium species (two diploids and two allotetraploid) were analyzed. Numts in four Gossypium nuclear genomes were detected by whole-genome alignment. Dot matrix analysis of mitochondrial vs nuclear genomes in G. raimondii $\left(D_{5}\right)$ show that there is a stretch of $\sim 598 \mathrm{~kb}(92.91 \%)$ of sequence that is nearly identical to that of the G. raimondii mitochondrial genome (Fig. 7a) in chromosome 1. This insertion is at least $99.80 \%$ identical to the mitochondrial genome, suggesting that the transfer event was very recent. The organization of the assembled mitochondrial genome differs from that of the mitochondrial DNA in the nucleus with an internal deletion (Fig. 7a), which might occur during or after transferring and represent an alternate isoform of the G. raimondii mitochondrial genome. In addition, G. hirsutum also has a nearly complete NUMT on chromosome A03 (Fig. 7c), and small to median-large fragments of mitochondrial DNA have been identified in three Gossypium species nuclear genomes (Fig. 7b-d), showing apparently sporadic fragmentation compared to G. raimondii. So much noise in Gossypium nuclear chromosomes of (Fig. 7b-d) are just repetitive derived elements. These results may be caused by the insertion of retrotransposon elements into mitochondrial DNA insertions that may contribute significantly to their fragmentation process in the other three nuclear genomes.

In addition, most numts had $>99 \%$ nucleotide identity to the homologous organelle sequences, so the lack of divergence in G. raimondii indicates that they must have been transferred to the nucleus recently. In order to estimate how old these insertions are, $\mathrm{p}$-distance rates and some estimates of rate/million years between mitochondrial sequences and numts were studied here. We have dated 20 larger NUMTs in G. raimondii (Additional file 5: Table S2), 16 larger NUMTs in G. arboreum (Additional file 6: Table S3), 15 larger NUMTs in G. hirsutum (Additional file 7: Table S4) and 12 larger NUMTs in G. barbadense (Additional file 8: Table S5). These data showed that the insertion time of NUMTs was close among one chromosome, but with big divergence between different chromosomes. For example, the different insertion time for five larger NUMTs in chromosome A03 of G. hirsutum (ranging from 0.33-1.03 MYA), with other chromosomes (insertion time ranging from 0.91-11.43 MYA) (Additional file 7: Table S4).

The p-distance of larger NUMTs ranged from 0 0.0009 in chromosome 01 of G. raimondii (Fig. 8a; Additional file 5: Table S2) and 0.0022 0.0069 in chromosome A03 of G. hirsutum (Fig. 8a; Additional file 7: Table S4). We have dated these larger NUMTs in chromosome 01 of G. raimondii with insertion time ranging from $0 \sim 0.13$ MYA 
Table 4 The numbers of edit sites in Gossypium mitochondrial protein-coding genes

\begin{tabular}{|c|c|c|c|c|c|c|c|c|c|c|}
\hline Genes & G. thurberi & G. davidsonii & G. raimondii & G. trilobum & G. arboreum & G. hirsutum & G. barbadense & G. tomentosum & G. mustelinum & G. darwinii \\
\hline atp1 & 6 & 6 & 6 & 6 & 6 & 6 & 6 & 6 & 6 & 6 \\
\hline atp4 & 11 & 11 & 11 & 11 & 11 & 11 & 11 & 11 & 11 & 11 \\
\hline atp6 & 9 & 9 & 9 & 9 & 9 & 9 & 9 & 9 & 9 & 9 \\
\hline atp8 & 3 & 3 & 3 & 3 & 3 & 3 & 3 & 3 & 3 & 3 \\
\hline atp9 & 6 & 6 & 6 & 6 & 6 & 6 & 6 & 6 & 6 & 6 \\
\hline $\mathrm{ccmB}$ & 29 & 29 & 29 & 29 & 29 & 29 & 29 & 29 & 29 & 29 \\
\hline $\mathrm{ccmC}$ & 24 & 24 & 24 & 24 & 24 & 24 & 24 & 24 & 24 & 24 \\
\hline $\mathrm{ccmFC}$ & 13 & 13 & 13 & 13 & 13 & 13 & 13 & 13 & 13 & 13 \\
\hline $\mathrm{ccmFn}$ & 31 & 31 & 31 & 31 & 31 & 31 & 31 & 31 & 31 & 31 \\
\hline$c o b$ & 9 & 9 & 9 & 9 & 9 & 9 & 9 & 9 & 9 & 9 \\
\hline $\cos 1$ & 16 & 16 & 16 & 16 & 16 & 16 & 16 & 16 & 16 & 16 \\
\hline $\cos 2$ & 12 & 12 & 12 & 12 & 12 & 12 & 12 & 12 & 12 & 12 \\
\hline $\cos 3$ & 5 & 5 & 5 & 5 & 5 & 5 & 5 & 5 & 5 & 5 \\
\hline matR & 15 & 15 & 15 & 15 & 15 & 15 & 15 & 15 & 15 & 15 \\
\hline$m t t B$ & 25 & 25 & 25 & 25 & 25 & 25 & 25 & 25 & 25 & 25 \\
\hline nad1 & 19 & 19 & 19 & 19 & 19 & 19 & 19 & 19 & 19 & 19 \\
\hline nad2 & 25 & 25 & 25 & 25 & 25 & 25 & 25 & 25 & 25 & 25 \\
\hline nad3 & 13 & 13 & 13 & 13 & 13 & 12 & 13 & 13 & 13 & 13 \\
\hline nad4 & 29 & 29 & 29 & 29 & 29 & 29 & 29 & 29 & 29 & 29 \\
\hline nad4L & 11 & 11 & 11 & 11 & 11 & 11 & 11 & 11 & 11 & 11 \\
\hline nad5 & 24 & 24 & 24 & 24 & 24 & 24 & 24 & 24 & 24 & 24 \\
\hline nad6 & 12 & 12 & 12 & 12 & 12 & 12 & 12 & 12 & 12 & 12 \\
\hline nad7 & 23 & 23 & 23 & 23 & 23 & 23 & 23 & 23 & 23 & 23 \\
\hline nadg & 8 & 8 & 8 & 8 & 8 & 8 & 8 & 8 & 8 & 8 \\
\hline rpl2 & 4 & 4 & 4 & 4 & 4 & 4 & 4 & 4 & 4 & 4 \\
\hline rpl5 & 10 & 10 & 10 & 10 & 10 & 10 & 10 & 10 & 10 & 10 \\
\hline rp/10 & 4 & 4 & 4 & 4 & 4 & 4 & 4 & 4 & 4 & 4 \\
\hline$r p / 16$ & 6 & 6 & 6 & 6 & 6 & 6 & 6 & 6 & 6 & 6 \\
\hline rps3 & 10 & 10 & 10 & 10 & 10 & 10 & 10 & 10 & 10 & 10 \\
\hline rps4 & 17 & 17 & 17 & 17 & 17 & 17 & 17 & 17 & 17 & 17 \\
\hline rps7 & 2 & 2 & 2 & 2 & 2 & 2 & 2 & 2 & 2 & 2 \\
\hline rps 10 & 5 & 5 & 5 & 5 & 5 & 5 & 5 & 5 & 5 & 5 \\
\hline rps 12 & 4 & 4 & 4 & 4 & 4 & 4 & 4 & 4 & 4 & 4 \\
\hline rps 14 & 3 & 3 & 3 & 3 & 3 & 3 & 3 & 3 & 3 & 3 \\
\hline sdh3 & 4 & 4 & 4 & 4 & 4 & 4 & 4 & 4 & 4 & 4 \\
\hline sdh4 & 4 & 4 & 4 & 4 & 4 & 4 & 4 & 4 & 4 & 4 \\
\hline Total & 451 & 451 & 451 & 451 & 451 & 450 & 451 & 451 & 451 & 451 \\
\hline Edits/gene & 12.5 & 12.5 & 12.5 & 12.5 & 12.5 & 12.5 & 12.5 & 12.5 & 12.5 & 12.5 \\
\hline
\end{tabular}

(Fig. 8b; Additional file 5: Table S2), and from 0.33 1.03 MYA in chromosome A03 of G. hirsutum (Fig. 8b; Additional file 7: Table S4). These results revealed that two nearly full length insertion events in G. raimondii (Chr01, Fig. 7a) and G. hirsutum (chromosome A03, Fig. 7c) occurred recently.

\section{Discussion}

From the perspective of divergence, Gossypium originated from a common ancestor approximately ten million years ago and an allopolyploidization event occurred approximately 1.5 million years ago $[35,36]$. Plant mitochondrial genomes have experienced myriad synteny-disrupting 


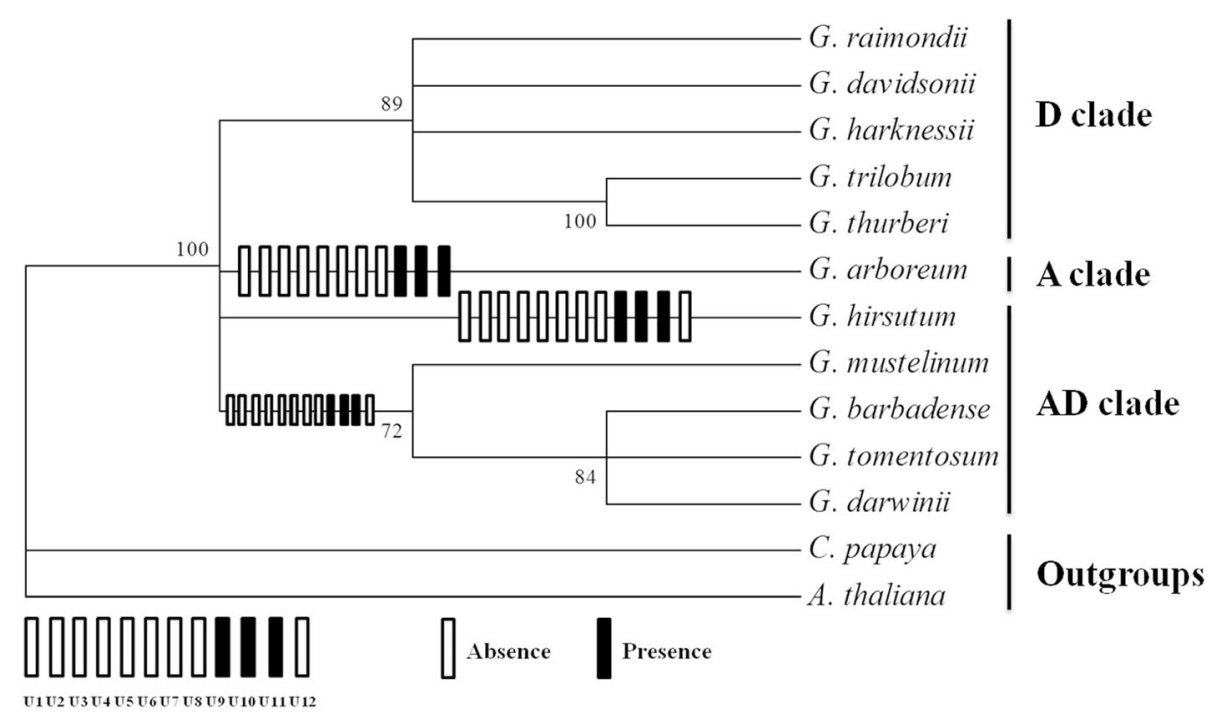

Fig. 5 Maximum likelihood (ML) phylogenetic tree of ten Gossypium species was constructed based on nucleotide sequences of 36 mitochondrial genes. Bootstrap values for all major divergences were high (>70\%) on the corresponding nodes. The hollow or black bars represent unique present or absent segments in ten Gossypium mitogenomes

rearrangements even over a very short evolutionary timescale. Like most angiosperm mitogenomes abundant in repeat sequences with larger repeats mediating recombination at moderate to high frequency [19, 77], these recombination events generated multiple mito-genomic arrangements differed in Gossypium genome groups, which may be largely caused by both larger repeats and some key InDels or SVs during evolution, and quickly eroded synteny even among closely related plants $[8,72,73,78]$.

These cotton mitochondrial genomes diverged much, as indicated by the InDels events unique to A genome species, D and AD groups, respectively. All these structural variants (SVs) are located in intergenic regions of

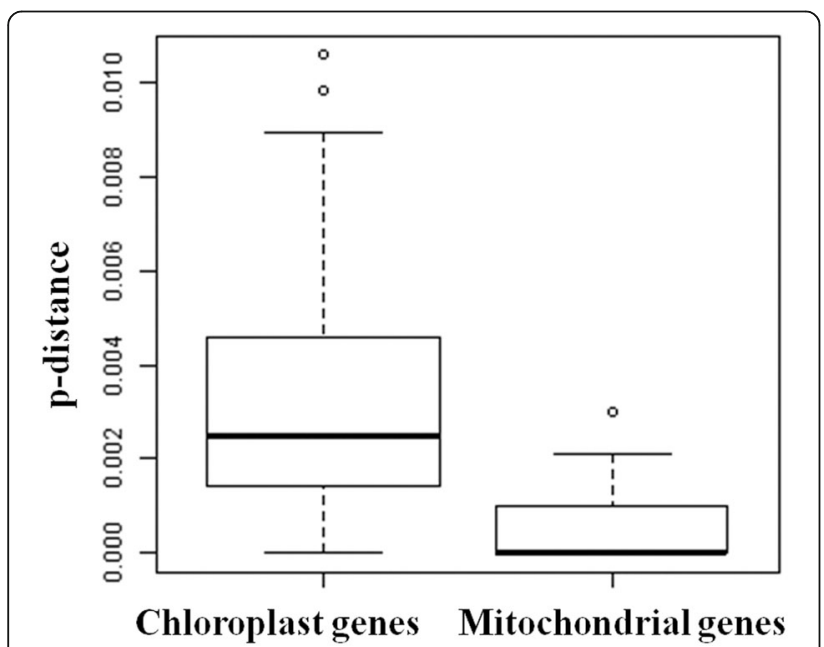

Fig. 6 Distribution of p-distances from 78 chloroplast and 36 mitochondrial protein-coding exons among 10 Gossypium species mitogenomes. Some of them overlapped with their breakpoints and junctions occurring in repetitive and homologous genomic regions. And insertions or deletions were mostly generated from crossovers of repetitive regions or homologous regions [79].

There existed apparent inversions and translocations, which can offer clues to explain gene order differences of mitogenomes between different Gossypium groups. For examples, the mode of gene order changed by inversions and/or translocations was presented in early land plant mitochondrial genomes evolution of bryophytes [80] as well as rapidly rearranged mitochondrial genomes of vascular plants [71-73, 81, 82]. Apart from apparent rearranged mitogenome organizations in diploid and allotetraploid Gossypium, mitochondrial genome rearrangements have also been detected in diploid and allotetraploid species of Brassica [74, 83]. Generally, apparent variations in the mitogenome structures were always tested to be associated with cytoplasmic male sterility (CMS) and its maintainer lines [71-73], thus a new mitochondrial gene was produced by recombination and conferred CMS with its encoded protein interacted with the nuclear encoded mitochondrial protein to cause a detrimental interaction [43]. However, no genome rearrangement or large indel segments variations compared with mitogenomes of other $\mathrm{D}$ species, implying that the mitochondrial CMS-associated gene in cotton may function with different mechanism.

In addition, RNA-editing sites in Gossypium may not be in charge of cytoplasmic male sterility in $\mathrm{D}_{8}$ cotton. RNA editing events have been compared in eight mitochondrial genes (atp1, atp4, atp6, atp8, atp9, and cox1, 


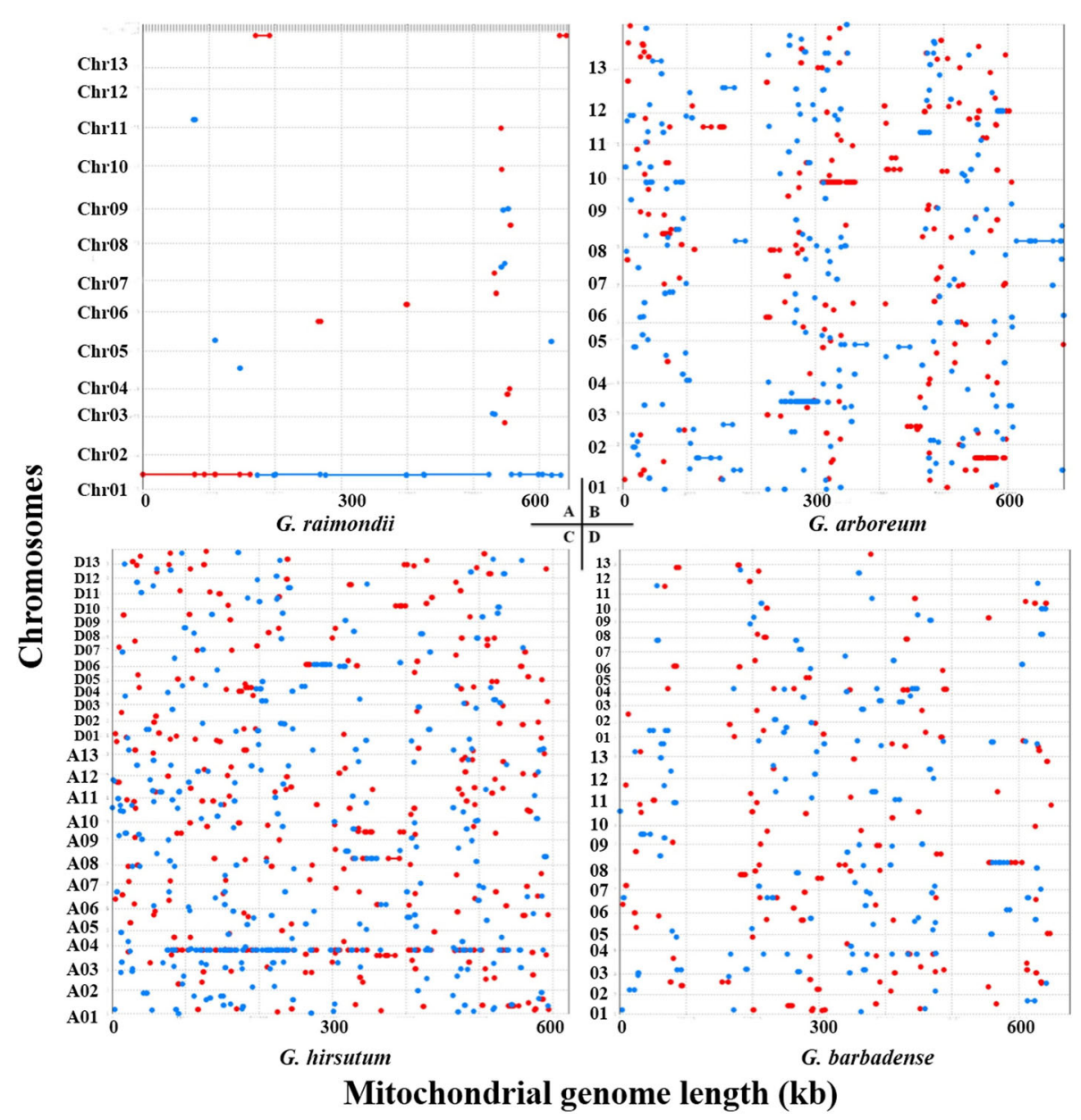

Fig. 7 Mitochondrial DNAs insertions into four Gossypium nuclear genomes detected by whole-genome alignment. The results were filtered to select only those alignments which comprise the one-to-one mapping between reference and query, and then display a dotplot of the selected alignments. The red and blue lines refer positive and reverse matches, respectively. a: Dot matrix analysis of numts in Gossypium raimondii $\left(D_{5}\right)$ nuclear genome performed using MUMmer (Delcher et al., 2002). b: Dot matrix analysis of numts in G. arboreum ( $A_{2}$ ) nuclear genome. c: Dot matrix analysis of numts in G. hirsutum $\left(A D_{1}\right)$ nuclear genome. d: Dot matrix analysis of numts in $G$. barbadense $\left(A D_{2}\right)$ nuclear genome

cox2, cox3) among CMS- $\mathrm{D}_{8}$ three lines in cotton [75]. Although the frequencies of RNA editing events between mtDNA genes were different, no differences between cotton cytoplasms that could account for the CMS phenotype or restoration. In view of these results, the complete mitogenome sequences will provide the useful data resources for targeting the CMS-related genes in G. trilobum $\mathrm{D}_{8}$ cotton in further studies.

As for MtDNAs insert into the nuclear chromosomes in Gossypium, Lin et al., (1999) and Stupar et al., (2001) also identified an intact mtDNA copy on chromosome 2 in the nucleus of Arabidopsis with more than 99\% identity, which proved this type of mitochondrion-to-nucleus migration event [84, 85]. Second, these mitochondrion-to-nucleus migrations proved to be the independent events after the divergence of the Gossypium progenitors. These genome changes within the diploid and allotetraploid Gossypium species is worthy of more attention in future studies.

\section{Conclusions}

Plants mitochondrial genomes are evolutionarily intriguing because of the highly conserved genic content and slow rates of genic sequence evolution $[18,82]$. These features contrasted sharply with the highly labile genomic structure, genome size, DNA repair mechanism and recombination induced by different types and origins of repeated sequences [82, 86-88]. Whole mitogenome sequences have been released in an ongoing process $[9,11,38,81,89]$, which provide information for dissecting the evolutionary modifications in these genomes, such as gene loss [88], sequence acquisitions or loss [9], multiple sequence rearrangements [73] and dynamic structure evolution $[38,39]$. Here, we presented six more cotton mitochondrial genomes, which showed apparently distinct divergence. Despite the short divergence time separating diploid and allotetraploid cotton species [35, 36], many of the hallmark features of mitochondrial genome evolution are evident, 


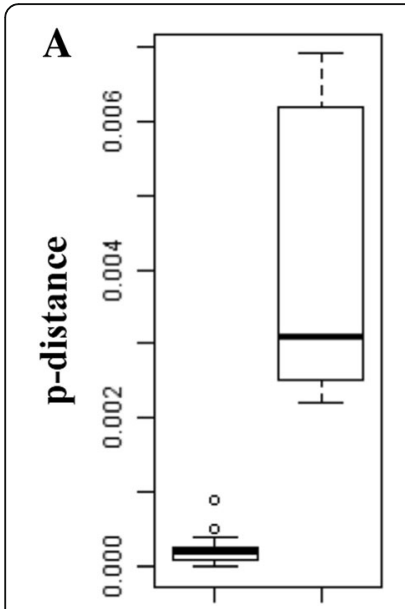

G. raimondii G. hirsutum

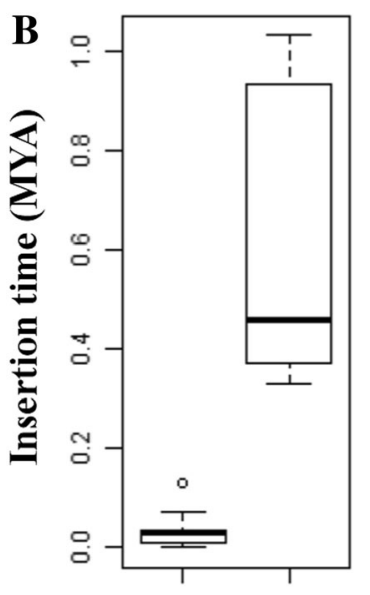

G. raimondii G. hirsutum
Fig. 8 p-distances (a) and estimated divergence time (b) of two recent nearly full length insertion in G. raimondii (Chr01, Fig. 7a) and G. hirsutum (Chr A03, Fig. 7c)

including differential genic content, genome rearrangements, inversion and translocation, gains/losses of multiple small and large repeats, presence/absence variations, and the mitogenome of G. trilobum $\mathrm{D}_{8}$ cotton for targeting CMS-associated gene. Comparative analyses illustrated that four of the outcomes are quite surprising, including: 1) how rapidly mitochondrial genome rearrangements occur within a single subspecies (diverged $~ 10$ mya), 2) how rapidly mitochondrial sequence segments are gained or lost, 3) RNA editing events were almost conserved in ten Gossypium mitogenomes, and 4) a previous unusual report of the integration of $93 \%$ of the mitochondrial genome of G. raimondii into chromosome 1 is confirmed with an estimation of insertion time 0.05 MYA. Increasing insight into the mechanisms and functional consequences of plant mitochondrial genome variation are expected to be helpful to elucidate the process of rapid evolutionary divergence mechanism between closely related mitochondrial genomes.

\section{Additional files}

Additional file 1: Figure S1. Genome maps of six diploid and allotetraploid Gossypium mitogenomes. Genes exhibited on the inside of outer circles are transcribed in a clockwise direction, while genes on the outside of outer circles are transcribed in a reverse direction. (JPEG 424 kb)

Additional file 2: Table S1. Gene contents of the six Gossypium mitogenomes. Note: Genes presented in multiple copies are denoted with a number (e.g., 2 or 3). (DOCX 18 kb)

Additional file 3: Figure S2. Gene order comparison among mitogenomes of Gossypium. Colored blocks represent regions of conserved gene clusters in the Gossypium genomes and genes in bold are located in the repeat regions. Rp/2 and atp 8 are shown in red bold to indicate that they are just close to or partially overlapped with the repeat sequences. (JPEG $1006 \mathrm{~kb}$ )
Additional file 4: Figure S3. Observed coverage of mapped paired-end reads supporting the existence of a small deletion ( $500 \mathrm{bp})$ in $\mathrm{G}$. arboreum compared to AD group species. IGV screenshot of the variability and coverage observed in ten samples of Gossypium sequence. Upper panel represent the unique sequences coordinates. There are five panels corresponding to the different Gossypium sequences. The track in each of these panels describes the density of read mapping or coverage depth. (JPEG $353 \mathrm{~kb}$ )

Additional file 5: Table S2. Nucleotide distances and divergence time (MYA) between mitochondrial sequences and corresponding numts in $G$. raimondii. Note: ${ }^{a}$ twenty numts represent the largest mitochondrial fragments transferred into the nuclear chromosomes in G. raimondii. (DOCX $17 \mathrm{~kb}$ )

Additional file 6: Table S3. Nucleotide distances and divergence time (MYA) between mitochondrial sequences and corresponding numts in G. arboreum. (DOCX $17 \mathrm{~kb}$ )

Additional file 7: Table S4. Nucleotide distances and divergence time (MYA) between mitochondrial sequences and corresponding numts in $G$. hirsutum. Note: ${ }^{a}$ fifteen numts represent the largest mitochondrial fragments transferred into the nuclear chromosomes in G. hirsutum. ${ }^{\mathrm{b}}$ represents five fragments from nearly full length mitochondrial fragments transferred into the nuclear A03 chromosomes in G. hirsutum (Fig. 7C). (DOCX 17 kb)

Additional file 8: Table S5. Nucleotide distances and divergence time (MYA) between mitochondrial sequences and corresponding numts in $G$. barbadense. Note: ${ }^{a}$ twelve numts represent the largest mitochondrial fragments transferred into the nuclear chromosomes in G. barbadense. (DOCX $16 \mathrm{~kb})$

\section{Abbreviations}

CMS: Cytoplasmic male sterility; G.: Gossypium; IGV: Integrative Genomics Viewer; Indels: Insertions and deletions; Mitogenome: Mitochondrial genome; mtDNA: Mitochondrial DNA; ORFs: Open reading frames; rRNAs: Ribosomal RNAs; tRNAs: Transfer RNAs

\section{Acknowledgments}

We are indebted to Dr. Shaoqing Li and late Prof. Yingguo Zhu (College of Life Sciences, Wuhan University, China) for helpful suggestions. We also thank Professor Jonathan F. Wendel (lowa State University, Ames, USA), Dr. Corrinne E. Grover (lowa State University, Ames, USA) and Professor Shu-Miaw Chaw (BRCAS, Taiwan, China) for helpful discussions. We are grateful to Dr. Kunbo Wang (Chinese Academy of Agricultural Sciences) for providing the seeds of wild Gossypium species used in present research.

\section{Funding}

This work was supported by grant from the National Natural Science Foundation of China (31671741) to J. Hua.

\section{Availability of data and materials}

Complete DNA sequences were deposited in GenBank database (KR736343 for $G$. thurberi $D_{1}, K R 736344$ for $G$. davidsonii $D_{3-d}, K R 736346$ for $G$. trilobum $\mathrm{D}_{8}, \mathrm{KX} 388135$ for $\mathrm{G}$. tomentosum $\mathrm{AD}_{3}, \mathrm{KX} 388136$ for $G$. mustelinum $\mathrm{AD}_{4}$ and $\mathrm{KX} 388137$ for $\mathrm{G}$. darwinii $\mathrm{AD}_{5}$ ). Other data sets supporting the results of this article are included within the article and its additional files.

\section{Authors' contributions}

ZWC assembled the mitochondrial genome, annotated the mitochondrial genomes, performed the data analysis and prepared the manuscript. HSN, HLP, LDZ and SSL attended the data analyses and discussion. YMW maintained the experimental platform and participated in the bench work. JPH designed the experiments, provided research platform, guided the research and revised the manuscript. All authors approved the final manuscript.

Ethics approval and consent to participate

Not applicable.

Consent for publication

Not applicable.

\section{Competing interests}

The authors declare that they have no competing interests. 


\section{Publisher's Note}

Springer Nature remains neutral with regard to jurisdictional claims in published maps and institutional affiliations.

\begin{abstract}
Author details
'Laboratory of Cotton Genetics, Genomics and Breeding /Key Laboratory of Crop Heterosis and Utilization of Ministry of Education/Beijing Key Laboratory of Crop Genetic Improvement, College of Agronomy and Biotechnology, China Agricultural University, Beijing 100193, China. ${ }^{2}$ Institute of Cash Crops, Hubei Academy of Agricultural Sciences, Wuhan, Hubei 430064, China. ${ }^{3}$ Department of Plant Science, School of Agriculture and Biology, Shanghai Jiao Tong University, Shanghai 200240, China.
\end{abstract}

Received: 10 October 2016 Accepted: 7 November 2017 Published online: 13 November 2017

\section{References}

1. Backert S, Nielsen BL, Borner T. The mystery of the rings: structure and replication of mitochondrial genomes from higher plants. Trends Plant Sci. 1997:2(12):477-83.

2. Chen Z, Zhao N, Li S, Grover CE, Nie H, Wendel JF, et al. Plant mitochondrial genome evolution and cytoplasmic male sterility. Crit Rev Plant Sci. 2017; 36(1):55-69.

3. Unseld M, Marienfeld JR, Brandt P, Brennicke A. The mitochondrial genome of Arabidopsis thaliana contains 57 genes in 366,924 nucleotides. Nat Genet. 1997;15(1):57-61.

4. Gualberto JM, Newton KJ. Plant mitochondrial genomes: dynamics and mechanisms of mutation. Annu Rev Plant Biol. 2017;68:225-52.

5. Oldenburg DJ, Bendich AJ. Size and structure of replicating mitochondrial DNA in cultured tobacco cells. Plant Cell. 1996;8(3):447-61.

6. Oldenburg DJ, Bendich AJ. Mitochondrial DNA from the liverwort Marchantia polymorpha: circularly permuted linear molecules, head-to-tail concatemers, and a 5' protein. J Mol Biol. 2001;310(3):549-62.

7. Bendich AJ. The size and form of chromosomes are constant in the nucleus, but highly variable in bacteria, mitochondria and chloroplasts. BioEssays. 2007;29(5):474-83

8. Sloan DB, Alverson AJ, Chuckalovcak JP, Wu M, McCauley DE, Palmer JD, et al. Rapid evolution of enormous, multichromosomal genomes in flowering plant mitochondria with exceptionally high mutation rates. PLoS Biol. 2012; 10(1):e1001241.

9. Rice DW, Alverson AJ, Richardson AO, Young GJ, Sanchez-Puerta MV, Munzinger J, et al. Horizontal transfer of entire genomes via mitochondrial fusion in the angiosperm Amborella. Science. 2013;342(6165):1468-73.

10. Alverson AJ, Rice DW, Dickinson S, Barry K, Palmer JD. Origins and recombination of the bacterial-sized multichromosomal mitochondrial genome of cucumber. Plant Cell. 2011;23(7):2499-513.

11. Skippington E, Barkman TJ, Rice DW, Palmer JD. Miniaturized mitogenome of the parasitic plant Viscum scurruloideum is extremely divergent and dynamic and has lost all nad genes. P Natl Acad Sci USA. 2015;112(27): E3515-24.

12. Kitazaki K, Kubo T. Cost of having the largest mitochondrial genome: evolutionary mechanism of plant mitochondrial genome. Journal of Botany. 2010;2010:1-12

13. Galtier N. The intriguing evolutionary dynamics of plant mitochondrial DNA. BMC Biol. 2011;9:61.

14. Bonen L. Mitochondrial genes leave home. New Phytol. 2006;172(3):379-81.

15. Bergthorsson U, Adams KL, Thomason B, Palmer JD. Widespread horizontal transfer of mitochondrial genes in flowering plants. Nature. 2003;424(6945): 197-201.

16. Rodriguez-Moreno L, Gonzalez VM, Benjak A, Marti MC, Puigdomenech P, Aranda MA, et al. Determination of the melon chloroplast and mitochondrial genome sequences reveals that the largest reported mitochondrial genome in plants contains a significant amount of DNA having a nuclear origin. BMC Genomics. 2011;12:424

17. Goremykin W, Lockhart PJ, Viola R, Velasco R. The mitochondrial genome of Malus domestica and the import-driven hypothesis of mitochondrial genome expansion in seed plants. Plant J. 2012;71(4):615-26.

18. Davila Jl, Arrieta-Montiel MP, Wamboldt Y, Cao J, Hagmann J, Shedge V, et al. Double-strand break repair processes drive evolution of the mitochondrial genome in Arabidopsis. BMC Biol. 2011;9:64.
19. Marechal A, Brisson N. Recombination and the maintenance of plant organelle genome stability. New Phytol. 2010;186(2):299-317.

20. Wendel JF, Cronn RC. Polyploidy and the evolutionary history of cotton. Adv Agron. 2003;78:139-86.

21. Wendel JF, Grover CE. Taxonomy and evolution of the cotton genus. In: Fang D, Percy R, editors. Cotton, Agronomy. Madison: Monograph 24, ASACSSA-SSSA; 2015.

22. Gallagher JP, Grover CE, Rex K, Moran M, Wendel JF. A New species of cotton from wake atoll, Gossypium stephensii (Malvaceae). Syst Bot 2017;42(1):115-123.

23. Wendel JF, Brubaker CL, Seelanan T. The origin and evolution of Gossypium. In: Stewart JM, Oosterhuis DM, Heitholt JJ, Mauney JR, editors. Physiology of cotton. Dordrecht: Springer Netherlands; 2010. p. 1-18.

24. Shendure J, Ji HL. Next-generation DNA sequencing. Nat Biotechnol. 2008; 26(10):1135-45.

25. Ansorge WJ. Next-generation DNA sequencing techniques. New Biotechnol. 2009;25(4):195-203.

26. Wang $K$, Wang Z, Li F, Ye W, Wang J, Song G, et al. The draft genome of a diploid cotton Gossypium raimondii. Nat Genet. 2012;44(10):1098-103.

27. Paterson $\mathrm{AH}$, Wendel JF, Gundlach $\mathrm{H}$, Guo H, Jenkins J, Jin D, et al. Repeated polyploidization of Gossypium genomes and the evolution of spinnable cotton fibres. Nature. 2012;492(7429):423-7.

28. Li F, Fan G, Wang K, Sun F, Yuan Y, Song G, et al. Genome sequence of the cultivated cotton Gossypium arboreum. Nat Genet. 2014:46(6):567-72.

29. Li F, Fan G, Lu C, Xiao G, Zou C, Kohel RJ, et al. Genome sequence of cultivated upland cotton (Gossypium hirsutum TM-1) provides insights into genome evolution. Nat Biotechnol. 2015;33(5):524-30.

30. Zhang T, Hu Y, Jiang W, Fang L, Guan X, Chen J, et al. Sequencing of allotetraploid cotton (Gossypium hirsutum L. acc. TM-1) provides a resource for fiber improvement. Nat Biotechnol. 2015;33(5):531-7.

31. Liu X, Zhao B, Zheng HJ, Hu Y, Lu G, Yang CQ, et al. Gossypium barbadense genome sequence provides insight into the evolution of extra-long staple fiber and specialized metabolites. Sci Rep-Uk. 2015:5:14139.

32. Yuan DJ, Tang ZH, Wang MJ, Gao WH, Tu LL, Jin X, et al. The genome sequence of Sea-Island cotton (Gossypium barbadense) provides insights into the allopolyploidization and development of superior spinnable fibres. Sci Rep-Uk. 2015:5:17662.

33. Lee SB, Kaittanis C, Jansen RK, Hostetler JB, Tallon $\sqcup$, Town CD, et al. The complete chloroplast genome sequence of Gossypium hirsutum: organization and phylogenetic relationships to other angiosperms. BMC Genomics. 2006:7:61.

34. Xu Q, Xiong GJ, Li PB, He F, Huang Y, Wang KB, et al. Analysis of complete nucleotide sequences of 12 Gossypium chloroplast genomes: origin and evolution of allotetraploids. PLoS One. 2012;7(8):e37128.

35. Chen Z, Feng K, Grover CE, Li P, Liu F, Wang Y, et al. Chloroplast DNA structural variation, phylogeny, and age of divergence among diploid cotton species. PLoS One. 2016;11(6):e0157183.

36. Chen Z, Grover CE, Li P, Wang Y, Nie H, Zhao Y, et al. Molecular evolution of the plastid genome during diversification of the cotton genus. Mol Phylogenet Evol. 2017;112:268-76.

37. Liu GZ, Cao DD, Li SS, AG S, Geng JN, Grover CE, et al. The complete mitochondrial genome of Gossypium hirsutum and evolutionary analysis of higher plant mitochondrial genomes. PLoS One. 2013;8(8):e69476.

38. Tang M, Chen Z, Grover CE, Wang Y, Li S, Liu G, et al. Rapid evolutionary divergence of Gossypium barbadense and G. hirsutum mitochondrial genomes. BMC Genomics. 2015;16:770.

39. Chen Z, Nie H, Grover CE, Wang Y, Li P, Wang M, et al. Entire nucleotide sequences of Gossypium raimondii and G. arboreum mitochondrial genomes revealed A-genome species as cytoplasmic donor of the allotetraploid species. Plant Biol. 2017;19(3):484-93.

40. Chen LT, Liu YG. Male sterility and fertility restoration in crops. Annu Rev Plant Biol. 2014;65:579-606

41. Sloan DB, Muller K, McCauley DE, Taylor DR, Storchova H. Intraspecific variation in mitochondrial genome sequence, structure, and gene content in Silene vulgaris, an angiosperm with pervasive cytoplasmic male sterility. New Phytol. 2012;196(4):1228-39.

42. Tang $H$, Zheng $X$, Li C, Xie $X$, Chen $Y$, Chen $L$, et al. Multi-step formation, evolution, and functionalization of new cytoplasmic male sterility genes in the plant mitochondrial genomes. Cell Res. 2017:27(1):130-46.

43. Luo D, Xu H, Liu Z, Guo J, Li H, Chen L, et al. A detrimental mitochondrialnuclear interaction causes cytoplasmic male sterility in rice. Nat Genet. 2013; 45(5):573-7. 
44. Horn R, Gupta KJ, Colombo N. Mitochondrion role in molecular basis of cytoplasmic male sterility. Mitochondrion. 2014;19:198-205.

45. Hu J, Huang WC, Huang Q, Qin XJ, CC Y, Wang LL, et al. Mitochondria and cytoplasmic male sterility in plants. Mitochondrion. 2014;19:282-8.

46. Li SS, Liu GZ, Chen ZW, Wang YM, Li PB, Hua JP. Construction and initial analysis of five Fosmid libraries of mitochondrial genomes of cotton (Gossypium). Chinese Sci Bull 2013;58(36):4608-4615.

47. Bolger AM, Lohse M, Usadel B. Trimmomatic: a flexible trimmer for Illumina sequence data. Bioinformatics. 2014;30(15):2114-20.

48. Zerbino DR, Birney E. Velvet: algorithms for de novo short read assembly using de Bruijn graphs. Genome Res. 2008;18(5):821-9.

49. Magoc T, Salzberg SLFLASH. Fast length adjustment of short reads to improve genome assemblies. Bioinformatics. 2011;27(21):2957-63.

50. Altschul SF, Gish W, Miller W, Myers EW, Lipman DJ. Basic local alignment search tool. J Mol Biol. 1990;215(3):403-10.

51. Li H, Durbin R. Fast and accurate long-read alignment with burrowswheeler transform. Bioinformatics. 2010;26(5):589-95.

52. Li H, Handsaker B, Wysoker A, Fennell T, Ruan J, Homer N, et al. The sequence alignment/map format and SAMtools. Bioinformatics. 2009;25(16):2078-9.

53. Lowe TM, Eddy SR. tRNAscan-SE: a program for improved detection of transfer RNA genes in genomic sequence. Nucleic Acids Res. 1997;25(5):955-64.

54. Delcher AL, Phillippy A, Carlton J, Salzberg SL. Fast algorithms for large-scale genome alignment and comparison. Nucleic Acids Res. 2002;30(11):2478-83.

55. Lohse M, Drechsel O, Bock R. OrganellarGenomeDRAW (OGDRAW): a tool for the easy generation of high-quality custom graphical maps of plastid and mitochondrial genomes. Curr Genet. 2007;52(5-6):267-74.

56. Krzywinski M, Schein J, Birol I, Connors J, Gascoyne R, Horsman D, et al. Circos: an information aesthetic for comparative genomics. Genome Res. 2009;19(9):1639-45.

57. Darling AE, Mau B, Perna NT. progressiveMauve: multiple genome alignment with gene gain, loss and rearrangement. PLoS One. 2010;5(6):e11147.

58. Robinson JT, Thorvaldsdottir H, Winckler W, Guttman M, Lander ES, Getz G, et al. Integrative genomics viewer. Nat Biotechnol. 2011;29(1):24-6.

59. Mower JP. The PREP suite: predictive RNA editors for plant mitochondrial genes, chloroplast genes and user-defined alignments. Nucleic Acids Res. 2009;37(Web Server issue):W253-9.

60. Katoh K, Standley DMMAFFT. Multiple sequence alignment software version 7: improvements in performance and usability. Mol Biol Evol. 2013;30(4):772-80.

61. Tamura K, Peterson D, Peterson N, Stecher G, Nei M, Kumar S. MEGA5: molecular evolutionary genetics analysis using maximum likelihood, evolutionary distance, and maximum parsimony methods. Mol Biol Evol. 2011;28(10):2731-9.

62. Chaw SM, Chang CC, Chen HL, Li WH. Dating the monocot-dicot divergence and the origin of core eudicots using whole chloroplast genomes. J Mol Evol. 2004;58(4):424-41.

63. Muse SV. Examining rates and patterns of nucleotide substitution in plants. Plant Mol Biol. 2000;42(1):25-43.

64. Gaut BS, Morton BR, MCCaig BC, Clegg MT. Substitution rate comparisons between grasses and palms: synonymous rate differences at the nuclear gene Adh parallel rate differences at the plastid gene rbcL. Proc Natl Acad Sci U S A. 1996;93(19):10274-9.

65. Richardson AO, Rice DW, Young GJ, Alverson AJ, Palmer JD. The "fossilized" mitochondrial genome of Liriodendron tulipifera: ancestral gene content and order, ancestral editing sites, and extraordinarily low mutation rate. BMC Biol. 2013;11:29.

66. Guo W, Grewe F, Fan W, Young GJ, Knoop V, Palmer JD, et al. Ginkgo and Welwitschia mitogenomes reveal extreme contrasts in gymnosperm mitochondrial evolution. Mol Biol Evol. 2016;33(6):1448-60.

67. Zhang J, Turley RB, Stewart JM. Comparative analysis of gene expression between $C M S-D_{8}$ restored plants and normal non-restoring fertile plants in cotton by differential display. Plant Cell Rep. 2008;27(3):553-61.

68. Suzuki H, Rodriguez-Uribe L, Xu J, Zhang J. Transcriptome analysis of cytoplasmic male sterility and restoration in CMS-D $D_{8}$ cotton. Plant Cell Rep. 2013:32(10):1531-42

69. Suzuki H, Yu J, Wang F, Zhang J. Identification of mitochondrial DNA sequence variation and development of single nucleotide polymorphic markers for CMS-D 8 in cotton. Theor Appl Genet. 2013;126(6):1521-9.

70. Wendel JF. New world tetraploid cottons contain old world cytoplasm. Proc Natl Acad Sci U S A. 1989;86(11):4132-6.

71. Shearman JR, Sangsrakru D, Ruang-Areerate P, Sonthirod C, Uthaipaisanwong P, Yoocha T, et al. Assembly and analysis of a male sterile rubber tree mitochondrial genome reveals DNA rearrangement events and a novel transcript. BMC Plant Biol. 2014;14:45.

72. Tanaka Y, Tsuda M, Yasumoto K, Yamagishi H, Terachi T. A complete mitochondrial genome sequence of Ogura-type male-sterile cytoplasm and its comparative analysis with that of normal cytoplasm in radish (Raphanus sativus L.). BMC Genomics 2012;13:352.

73. Allen JO, Fauron CM, Minx P, Roark L, Oddiraju S, Lin GN, et al. Comparisons among two fertile and three male-sterile mitochondrial genomes of maize. Genetics. 2007;177(2):1173-92.

74. Grewe F, Edger PP, Keren I, Sultan L, Pires JC, Ostersetzer-Biran O, et al. Comparative analysis of 11 Brassicales mitochondrial genomes and the mitochondrial transcriptome of Brassica oleracea. Mitochondrion. 2014;19: 135-43.

75. Suzuki H, JW Y, Ness SA, O'Connell MA, Zhang JFRNA. Editing events in mitochondrial genes by ultra-deep sequencing methods: a comparison of cytoplasmic male sterile, fertile and restored genotypes in cotton. Mol Gen Genomics. 2013;288(9):445-57.

76. Takenaka M, Verbitskiy D, van der Merwe JA, Zehrmann A, Brennicke A. The process of RNA editing in plant mitochondria. Mitochondrion. 2008;8(1):35-46.

77. Woloszynska M. Heteroplasmy and stoichiometric complexity of plant mitochondrial genomes-though this be madness, yet there's method in't. J Exp Bot. 2010;61(3):657-71.

78. Palmer JD, Herbon LA. Plant mitochondrial DNA evolves rapidly in structure, but slowly in sequence. J Mol Evol. 1988;28(1-2):87-97.

79. Carbonell-Caballero J, Alonso R, Ibanez V, Terol J, Talon M, Dopazo JA. Phylogenetic analysis of 34 chloroplast genomes elucidates the relationships between wild and domestic species within the genus Citrus. Mol Biol Evol. 2015;32(8):2015-35.

80. Liu Y, Xue JY, Wang B, Li L, Qiu YL. The mitochondrial genomes of the early land plants Treubia lacunosa and Anomodon rugelii: dynamic and conservative evolution. PLoS One. 2011;6(10):e25836.

81. Wu ZQ, Cuthbert JM, Taylor DR, Sloan DB. The massive mitochondrial genome of the angiosperm Silene noctiflora is evolving by gain or loss of entire chromosomes. P Natl Acad Sci USA. 2015;112(33):10185-91.

82. Mower, J., D. Sloan, and A. Alverson, 2012 Plant mitochondrial genome diversity: the genomics revolution, pp. 123-144 in Plant Genome Diversity Volume 1, edited by J. F. Wendel, J. Greilhuber, J. Dolezel and I. J. Leitch. Springer Vienna.

83. Yang J, Liu G, Zhao N, Chen S, Liu D, Ma W, et al. Comparative mitochondrial genome analysis reveals the evolutionary rearrangement mechanism in Brassica. Plant Biol. 2016;18(3):527-36.

84. Lin XY, Kaul SS, Rounsley S, Shea TP, Benito MI, Town CD, et al. Sequence and analysis of chromosome 2 of the plant Arabidopsis thaliana. Nature. 1999:402(6763):761-8.

85. Stupar RM, Lilly JW, Town CD, Cheng Z, Kaul S, Buell CR, et al. Complex mtDNA constitutes an approximate 620-kb insertion on Arabidopsis thaliana chromosome 2: implication of potential sequencing errors caused by largeunit repeats. Proc Natl Acad Sci U S A. 2001;98(9):5099-103.

86. Christensen AC. Plant mitochondrial genome evolution can be explained by DNA repair mechanisms. Genome Biology and Evolution. 2013;5(6):1079-86.

87. Christensen AC. Genes and junk in plant mitochondria-repair mechanisms and selection. Genome Biology and Evolution. 2014;6(6):1448-53.

88. Adams KL, Qiu YL, Stoutemyer M, Palmer JD. Punctuated evolution of mitochondrial gene content: high and variable rates of mitochondrial gene loss and transfer to the nucleus during angiosperm evolution. P Natl Acad Sci USA. 2002;99(15):9905-12.

89. Eberhard JR, Wright TF. Rearrangement and evolution of mitochondrial genomes in parrots. Mol Phylogenet Evol. 2016;94:34-46. 